\title{
Luminescence studies of Schiff base metal complex for OLED applications
}

\author{
Navneet Kumar Gondia*, Jyoti Priya and S. K. Sharma \\ Department of Applied Physics, Indian School of Mines, Dhanbad, Jharkhand 826004 \\ *Email: navneetkumar50.in@gmail.com
}

The efficiency of electroluminescent organic light-emitting devices can be enhanced by using Schiff base phosphorescent material as emissive layer. Phosphorescent materials offer a means of achieving improved light-emission efficiencies as emission may arise from both singlet and triplet states. Due to these reasons Iridium, Osmium and Platinum complexes are extensively studied as phosphorescent emitter owing to its high photoluminescence quantum yield for OLED applications. However, these metals are comparatively expensive and the researchers are in search of a low-cost alternative. In contrast to these, cost effective metals such as copper and zinc presents an easily accessible alternative and offers a new opportunity to develop emitting materials.

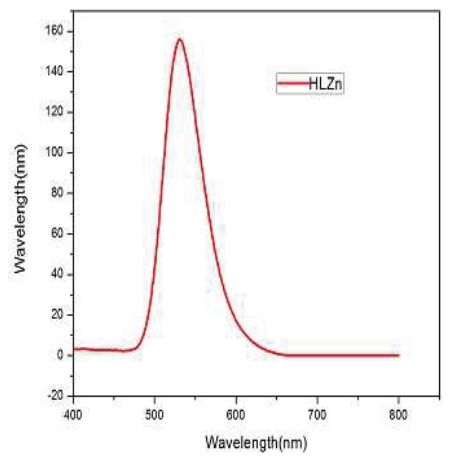

Figure 1: Emission spectra of zinc metal complex

First Copper complex for OLED was reported by Yuguang Ma and co-workers in 1999 as a new class of materials and after that research are still going on for the search of complexes with longer lifetime and high quantum yield. In the present work, a new Schiff base ligand along with its zinc metal complex was prepared for their suitable application as OLED emissive layer.

Formation of ligand \& metal complex was confirmed by NMR and FTIR spectroscopic techniques. Photoluminescence (PL) spectra were recorded keeping excitation wavelength fixed at $380 \mathrm{~nm}$. A strong intense emission peak was observed at $529 \mathrm{~nm}$ (Figure 1). HOMO/LUMO energy gap values were calculated using Gaussian Software and were found to be -0.222 and -0.057 for zinc metal complex. Color coordinates were calculated from CIE chromaticity diagram and were observed at $\mathrm{x}=0.283 \& \mathrm{y}=0.648$ (Figure 2). On the basis of luminescence studies and HOMO/LUMO values, the prepared metal complex may be considered as a good green light emitting phosphor for possible application as emissive layer in OLEDs.

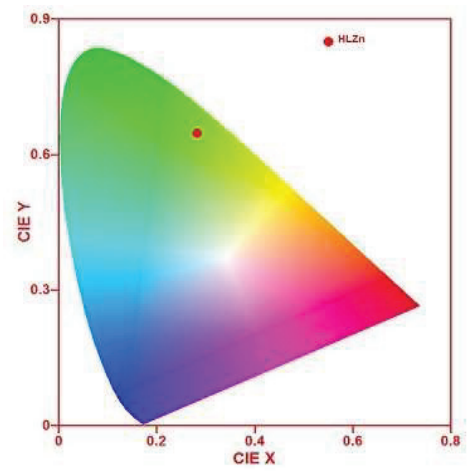

Figure 2: CIE Diagram of zinc metal complex

\section{References}

1. K. Quari, S. Bendia, J. Weiss, C. Bailly, Spectrochim. Acta 135 (2015) 624.

2. C.W. Tang, S.A. VanSlyke, Appl. Phys. Lett. 51 (1987) 913.

3. A.V. Metelitsa, A. S. Burlov, S. O. Bezuglyi, I. G. Borodkina, V. A. Bren, A. D. Garnovskii, and V. I. Minkin, Russ. Journal of Coord. Chem. 32 (2006) 894.

4.T. Yu, K. Zhang, Y. Zhao, C. Yang, H. Zhang, L. Qia, D. Fan, W. Dong, L.Chen, Y. Qiu, Inorg. Chim. Acta. 361 (2008) 233. 